\title{
Thermosensitive Poly(DHSe/PEG/PPG Urethane)-Based Hydrogel Extended Remdesivir Application in Ophthalmic Medication
}

\author{
Sennan $\mathrm{Xu}^{1,+}{ }^{1}$, Lingjie $\mathrm{Ke}^{2,+}$, Sichen Zhao ${ }^{1}$, Zhiguo $\mathrm{Li}^{2}{ }^{\mathbb{D}}$, Yang Xiao ${ }^{1}$, Yunlong $\mathrm{Wu}^{2}{ }^{2}$, Jie Ren ${ }^{1, *}$ and Yan Qiu ${ }^{1, *}$ \\ 1 Fujian Provincial Key Laboratory of Ophthalmology and Visual Science, Eye Institute, School of Medicine, \\ Xiamen University, Xiamen 361102, China; 24520191153693@stu.xmu.edu.cn (S.X.); \\ 24520191156040@stu.xmu.edu.cn (S.Z.); 24920172204654@stu.xmu.edu.cn (Y.X.) \\ 2 School of Pharmaceutical Sciences, Xiamen University, Xiamen 361102, China; \\ 32320191153429@stu.xmu.edu.cn (L.K.); lizhiguo@stu.xmu.edu.cn (Z.L.); wuyl@xmu.edu.cn (Y.W.) \\ * Correspondence: renjie_7912@xmu.edu.cn (J.R.); yanqiu@xmu.edu.cn (Y.Q.); Tel.: +86-137-7996-5700 (J.R.); \\ +86-137-7997-6753 (Y.Q.) \\ + These authors contributed equally to this work.
}

Citation: Xu, S.; Ke, L.; Zhao, S.; Li, Z.; Xiao, Y.; Wu, Y.; Ren, J.; Qiu, Y. Thermosensitive Poly(DHSe/PEG/PPG

Urethane)-Based Hydrogel Extended Remdesivir Application in

Ophthalmic Medication. Pharmaceutics 2022, 14, 50. https://doi.org/10.3390/ pharmaceutics 14010050

Academic Editor: Marta

González-Álvarez

Received: 16 November 2021 Accepted: 8 December 2021

Published: 27 December 2021

Publisher's Note: MDPI stays neutral with regard to jurisdictional claims in published maps and institutional affiliations.

Copyright: () 2021 by the authors. Licensee MDPI, Basel, Switzerland. This article is an open access article distributed under the terms and conditions of the Creative Commons Attribution (CC BY) license (https:// creativecommons.org/licenses/by/ $4.0 /)$.

\begin{abstract}
The spread of infection with severe acute respiratory syndrome coronavirus 2 (SARS-CoV-2) caused the coronavirus disease 2019 (COVID-19) outbreak beginning in March 2020. Currently, there is a lack of suitable dose formulations that interrupt novel coronavirus transmission via corneal and conjunctival routes. In the present study, we developed and evaluated a thermosensitive gelling system based on a selenium-containing polymer for topical ocular continuous drug release. In detail, di-(1-hydroxylundecyl) selenide (DHSe), poly(ethylene glycol) (PEG), and poly(propylene glycol) (PPG) were polymerized to form poly(DHSe/PEG/PPG urethane). The polymer was used to carry poorly water-soluble remdesivir (RDV) at room temperature to form the final thermosensitive in situ gel, which exhibited a typical sol-gel transition at $35^{\circ} \mathrm{C}$. The formed polymer was further characterized by rheology, thermology, and scanning electron microscopy. In vitro release studies and in vivo retention and penetration tests indicated that the thermogel provided the prolonged release of RDV. The RDV-loaded in situ gel was proven to be non-biotoxic against human corneal epithelial cells, with good ocular tolerance and biocompatibility in rabbit eyes.
\end{abstract}

Keywords: thermosensitive hydrogel; remdesivir; ophthalmic drug delivery; ocular retention; ocular irritation

\section{Introduction}

The ongoing coronavirus disease 2019 (COVID-19) pandemic was caused by the severe acute respiratory syndrome coronavirus 2 (SARS-CoV-2), a $\beta$-coronavirus within the family Coronaviridae [1]. According to the World Health Organization and Johns Hopkins University dashboard, the global COVID-19 case number stood at approximately 270 million and the death toll was more than 5.3 million by the end of December 2021 (https:/ / www.arcgis.com/apps/dashboards, accessed on 12 December 2021). The nature of the spread of respiratory viruses is typically associated with respiratory droplets, aerosol transmission, and physical contact with respiratory secretions [2,3]. The potential of SARS-CoV-2 to infect the ocular epithelia and mucosa has been highlighted through ocular surface exposure to pollutants and the virus [4]. Considering that the ocular tissues exhibit an anatomical proximity to the respiratory tract, viruses can lead to ocular complications and further cause acute respiratory infection $[5,6]$.

Documented reports of the distribution of angiotensin-converting enzyme 2 (ACE2) receptors and transmembrane protease serine 2 (TMPRSS2) protein in ocular tissues brought insights into the potential of SARS-CoV-2 to invade the anterior eye segment [6,7]. The susceptible population may be exposed to COVID-19 by touching the eyes with possibly 
contaminated hands. The contaminated tears passing down through the nasolacrimal duct to the nasal epithelium also serve as a route for ocular infection. Altogether, these findings shed new light on a possible route for infection by SARS-CoV-2, but this pathway is difficult to protect with ordinary external devices such as masks [8,9].

Remdesivir (RDV) is a broad-spectrum antiviral medication developed by Gilead Sciences (Foster, USA) [10]. The in vitro antiviral effects of RDV are supported by multiple trials [11,12]. On 22 October 2020, the US Food and Drug Administration (FDA) approved Veklury (RDV-loaded cyclodextrin) for the treatment of coronavirus infection. Several formulations (e.g., oral preparation) of RDV, and its parent nucleoside GS-441524, have been projected to expand RDV applications [13]. However, to our knowledge, the application of RDV in ophthalmology has not been reported. The development of RDV eye drops is of benefit to the susceptible population in case of occupational and accidental ocular exposure to SARS-CoV-2.

Ocular application of RDV has proven difficult due to its limited aqueous solubility. It is not possible to enhance the solubility using a heating process because RDV is unstable at high temperatures. Conventional ophthalmic formulations (e.g., eye drops and ointments) suffer from very short retention times at the corneal surface site, which results in poor ocular bioavailability [14]. To provide the possibility to address the abovementioned problems, a thermosensitive hydrogel with great biocompatibility, low toxicity, mucoadhesiveness, and excellent ocular tolerance is proposed in this paper. A thermosensitive triblock copolymer hydrogel containing selenol was developed because selenium acts as an essential trace element to protect the cells of the ocular surface $[15,16]$. To assess the potential feasibility of the RDV-loaded poly(DHSe/PEG/PPG urethane) thermogel as an ocular delivery system, optical transparency, rheological parameters, thermal properties, and sustained-release behavior were thoroughly characterized. A biocompatibility evaluation in human corneal epithelial cells (HCECs) and in rabbit eyes of the RDV-loaded thermogels was performed to verify the safety of the thermogel. Finally, the thermogel exhibited an increased contact time between the eye surface and enhanced drug permeation into the eye compartments, leading to better control of SARS-CoV-2 infection via the ocular route.

\section{Materials and Methods}

\subsection{Materials}

Di-(1-hydroxylundecyl) selenide (DHSe), polyethylene glycol (PEG), polypropylene glycol (PPG), 1,6-hexamethylene diisocyanate (HMDI), dibutyltin dilaurate (DBT), and paraplast plus were supplied by Sigma-Aldrich (St. Louis, MO, USA). Dulbecco's Modified Eagle's Medium/Ham's F-12 Medium (DMEM/F12) cell culture medium and fetal bovine serum were supplied by Life Technologies (Carlsbad, CA, USA), while 4,6-diamidino-2phenylindole (DAPI) and phosphate-buffered solution (PBS) were obtained from Beyotime Biotechnology (Shanghai, China). MD25-3.5 dialysis membranes (molecular weight cut-off $3.5 \mathrm{kDa}$ ) were purchased from Viskase Companies, Inc. (Chicago, IL, USA). A hematoxylineosin (H\&E) staining kit was obtained from KeyGEN BioTECH (Nanjing, China) and methanol (HPLC-grade) from Thermofisher (Waltham, MA, USA).

\subsection{Synthesis of Poly(DHSe/PEG/PPG Urethane)}

Poly(DHSe/PEG/PPG urethane) was synthesized by a modified method developed by our group [17]. The detailed synthetic procedure was as follows. Briefly, $10.0 \mathrm{~g}$ of PEG, PPG oligomers (PEG/PPG feed at 3/1 for DHSe-31 and PEG/ PPG feed at 1/1 for DHSe-11), and $0.05 \mathrm{~g}$ of DHSe-diol were mixed and then dried under vacuum at $40{ }^{\circ} \mathrm{C}$ overnight. The moisture content was then removed by azeotropic distillation with $200 \mathrm{~mL}$ anhydrous toluene. When the flask was placed in an oil bath at $75^{\circ} \mathrm{C}$ under argon, $0.913 \mathrm{~g}$ HMDI and two drops of DBT were added. The mixture was further stirred for $48 \mathrm{~h}$. After the preceding steps were completed, the collected copolymer was precipitated in ether and further purified via dialysis using a cut-off membrane. The resultant polyurethane was obtained through a freeze-drying process [18]. 


\subsection{Molecular Characterization}

Molecular characterization was performed using ${ }^{1} \mathrm{H}$ NMR spectra and gel permeation chromatography (GPC). ${ }^{1} \mathrm{H}$ NMR spectra were measured in $\mathrm{CDCl}_{3}$ solvent using JEOL $500 \mathrm{MHz}$ NMR spectrometers (JEOL USA, Peabody, MA, USA). GPC assay was performed at $40{ }^{\circ} \mathrm{C}$ on a Viscotek GPCmax module (Malvern Panalytical, Malvern, UK) equipped with two series-connected Phenogel ${ }^{\mathrm{TM}}$ columns $\left(10^{3}\right.$ and $\left.10^{5} \AA, 300 \times 7.80 \mathrm{~mm}\right)$. The eluent of GPC was tetrahydrofuran, and the flow rate was set at $1.0 \mathrm{~mL} / \mathrm{min}$. GPC profile was acquired with poly(methyl methacrylate), in a Viscotek refractive index detector.

\subsection{Determination of Thermal Properties}

The thermal properties were determined using differential scanning calorimetry (DSC) and thermogravimetric analysis (TGA). DSC assay was performed using a PDSC-Q100 calorimeter (TA Instruments, New Castle, DE, USA). Samples were heated in a stream of nitrogen, ranging from $-80{ }^{\circ} \mathrm{C}$ to $120^{\circ} \mathrm{C}$ (heating rate of $20^{\circ} \mathrm{C}$ per minute). TGA assay was conducted on a TGA Q500 analyzer (TA Instruments, New Castle, USA) under nitrogen at a heating rate of $20^{\circ} \mathrm{C}$ per minute from room temperature to $800^{\circ} \mathrm{C}$. Glass transition temperature $\left(T_{\mathrm{g}}\right)$ and decomposition temperature $\left(T_{\mathrm{d}}, 5 \%\right.$ weight loss) were calculated.

\subsection{Determination of Rheological Properties}

The rheological properties of the hydrogels were estimated in steady-state and dynamic modes using a DHR-3 rheometer (TA Instruments, New Castle, USA). Oscillatory amplitude sweeps were conducted at a frequency of $1 \mathrm{~Hz}$ and the frequency sweeps were conducted at a strain of $1 \%$. All tests were performed at $37.0^{\circ} \mathrm{C}$. Storage modulus $\left(G^{\prime}\right)$ and loss modulus $\left(G^{\prime \prime}\right)$ were measured as functional parameters.

\subsection{Determination of Sol-Gel Phase Transition}

Sol-gel phase transition was determined using an inverting vial method and a stirrer method in increments of $2{ }^{\circ} \mathrm{C}$ per minute $[19,20]$. A series of different concentrations ( 2 to $20 w / v \%$ ) of poly(DHSe/PEG/PPG urethane) copolymer systems were prepared. The inverting vial test consisted of equilibration at $4{ }^{\circ} \mathrm{C}$, followed by immersion for $5 \mathrm{~min}$ in a water bath. Gelation temperature (GT) was defined if the gel was firm enough over $1 \mathrm{~min}$ after inversion. As for the stirrer method, a magnetic bar and $5 \mathrm{~mL}$ of sample solution were placed into a $25 \mathrm{~mL}$ transparent vial. A mercury thermometer was kept perpendicularly positioned $3 \mathrm{~mm}$ from the bottom. The solution was heated with continuous stirring of 100 rounds per minute. The temperature at which the magnetic bar stopped turning was recorded as GT. In addition, the minimum gelation concentration (MGC) was defined as the minimum copolymer concentration at which the gelation behavior could be observed.

\subsection{Cryo-Scanning Electron Microscopy}

The microscopic appearance of the hydrogel was observed using a Quanta ${ }^{\text {TM }} 450$ FEG high-resolution SEM (FEI Company, Hillsboro, OR, USA), which was equipped with a PP3000T preparation and a cryo-transfer system (Quorumtech, Lewes, UK). During the cryo-scanning electron microscopy (Cryo-SEM) procedure, a small piece of the hydrogel sample (ca $2 \mathrm{~mm} \times 2 \mathrm{~mm} \times 2 \mathrm{~mm}$ ) was immersed in liquid nitrogen for $30 \mathrm{~s}$ and then transferred to the cryogenic reparation chamber using the cryo-preparation and transfer system. The frozen specimens were cut into thin sections for SEM observation, followed by automatic sublimation $\left(-90{ }^{\circ} \mathrm{C}, 10 \mathrm{~min}\right)$ and sputtering $(10 \mathrm{~mA}, 60 \mathrm{~s})$. Hydrogel morphology was observed in low-vacuum mode at an accelerating voltage of $5 \mathrm{kV}$; the cooling stage was set at $-140{ }^{\circ} \mathrm{C}$.

\subsection{Preparation of RDV-Loaded Poly(DHSe/PEG/PPG Urethane) Hydrogel}

Synthesis of RDV-loaded poly(DHSe/PEG/PPG urethane), namely $\mathrm{RDV} /$ poly(DHSe/PEG/PPG urethane), was performed using a two-step method at $4{ }^{\circ} \mathrm{C}$. Five or $10 \mathrm{mg}$ RDV and $10 \mathrm{mg}$ poly(DHSe/PEG/PPG urethane) were weighed into $300 \mu \mathrm{L}$ 
tetrahydrofuran (THF) to form final $1 \mathrm{mg} / \mathrm{mL}$ and $2 \mathrm{mg} / \mathrm{mL}$ RDV-loaded hydrogels. The mixture was then added into $700 \mu \mathrm{L}$ deinonized water using a slow drip with sonication. THF was then volatilized overnight, and equal volume deionized water was readded to form RDV-loaded micelles (Reagent A). Meanwhile, $800 \mu \mathrm{L}$ aqueous solution of $3 w / v \%$ poly(DHSe/PEG/PPG urethane) was equilibrated at $4{ }^{\circ} \mathrm{C}$ to obtain Reagent B. Finally, $200 \mu \mathrm{L}$ reagent $\mathrm{A}$ was mixed with reagent $\mathrm{B}$ with stirring and sonication at $4{ }^{\circ} \mathrm{C}$.

\subsection{Determination of Light Transmittance}

The optical transmittance of the hydrogel was estimated by comparing the $\mathrm{RDV} /$ poly(DHSe/PEG/PPG urethane) with RDV-loaded poloxamer (brand name Pluronic) hydrogel at $4{ }^{\circ} \mathrm{C}$ and $37^{\circ} \mathrm{C}$. The samples were first observed after being dripped onto a letter "A" card. Photographs were obtained using an Eclipse E400 camera (Nikon, Kyoto, Japan). The absorbance of all samples was then determined by continuous scanning within 380-780 nm range using an ultraviolet spectrophotometer (UV-2600, Shimadzu, Kyoto, Japan) and the results were combed with Shimadzu UVprobe2.42 software.

\subsection{In Vitro Drug Release Study}

RDV-loaded cyclodextrin inclusion complex was prepared as a control before the drug release study. Literature methods were followed as references [21,22]. The in vitro release profiles of RDV from the thermogels and cyclodextrin inclusion complexes were measured in Meilubio artificial tears (Dalian, China) at $35^{\circ} \mathrm{C}$. Briefly, $200 \mu \mathrm{L}$ of RDV-loaded thermogel and cyclodextrin inclusion compound (both $1 \mathrm{mg} / \mathrm{mL}$ ) were sealed into an MD25-3.5 dialysis membrane. Then, $2 \mathrm{~mL}$ of artificial tears was used as release medium. At predetermined time points, $1 \mathrm{~mL}$ of release medium was extracted, and $1 \mathrm{~mL}$ of artificial tears was replenished to maintain the volume. Cumulative release (\%) profile of RDV was calculated using the Agilent 1200 HPLC system (Agilent Poroshell 120 EC-C18, $4.6 \times 150 \mathrm{~mm}$ i.d., $4 \mu \mathrm{m}$ ) with a mobile phase of $0.05 \%$ TFA in $\mathrm{H}_{2} \mathrm{O} /$ acetonitrile $(50: 50 \mathrm{v} / \mathrm{v})$ at $254 \mathrm{~nm}$. The column temperature was $30{ }^{\circ} \mathrm{C}$ and the injection volume was set at $10 \mu \mathrm{L}$. The total run time was $31.0 \mathrm{~min}$ with a flow rate of $1.0 \mathrm{~mL} / \mathrm{min}$; the peak at around 18.5 min was assigned to RDV.

\subsection{Determination of Precorneal Fluorescent Dye Retention}

Fluorescein sodium cyclodextrin solution and poly(DHSe/PEG/PPG urethane) containing fluorescein sodium were used to determine the fluorescent dye retention. Briefly, $20 \mu \mathrm{L}$ fluorescein-containing eye drops was topically instilled twice to the ocular surface of rabbit eyes. The rabbits could blink as normal. Subsequently, the cornea and conjunctiva were imaged at predetermined points $(1,15,30$, and $60 \mathrm{~min})$ by an experienced oculist with a slit-lamp microscope with blue fluorescence (Leica, Wetzlar, Germany). The animal experiment in rabbits was approved in June 2019 by the Experimental Animal Ethics Committee of Xiamen University (IACUC: xmulac20190063).

\subsection{Imaging of Optical Coherence Tomography}

Corneal retention after topical administration of various formulations was determined using optical coherence tomography (OCT). C57BL/6 mice (female, 6-8 weeks of age) were anesthetized using 1\% Sumianxin-II (Shengda, Dunhua, China). OCT images were captured using a telecentric lens and an Optoprobe isOCT system (Optoprobe Science, Burnaby, BC, Canada). All images were obtained using volume scanning mode (volume size: width $=2048$, height $=600$, frames $=600$; data process: $\mathrm{C} 2=-43$, Fcoeff $=0.3$, $\max$ $\mathrm{Val}=40, \min \mathrm{Val}=20 ; \mathrm{XR}-\mathrm{YR}=2500 \mathrm{mV} \times 2500 \mathrm{mV} ; \mathrm{d} 1 / \mathrm{d} 2 / \mathrm{d} 3=100 / 10 / 5)$. After topical administration of $10 \mu \mathrm{L}$ of the eye drops and manual eyelid blinking, OCT images were obtained at predetermined points. 


\subsection{Measurement of RDV Penetration in Ocular Tissues}

After $20 \mu \mathrm{L}$ of RDV-containing drops was administered twice to the ocular surface in $1 \mathrm{~min}$, the rabbits were euthanized and corneas, conjunctiva, and aqueous fluid were collected [23]. All harvested tissues were weighed and stored at $-80^{\circ} \mathrm{C}$ for $1 \mathrm{~h}$ before ultra-performance liquid chromatography combined with tandem mass spectrometry (UPLC-MS/MS) analysis [24].

Before extraction, the samples were homogenized in $500 \mu \mathrm{L}$ distilled deionized water using a KZ-III-F Tissue Lyser (Servicebio, Wuhan, China). RDV was extracted from the homogenates using $1 \mathrm{~mL}$ of cold methanol containing $100 \mathrm{ng} / \mathrm{mL}$ of the internal standard substance (ISS). The ISS SML-2875, a nucleic acid analogue, was a generous gift from Prof. Zheng Xiao (Xiamen University, Xiamen, China). The solution was then centrifuged at $12,000 \mathrm{rpm}$ and the supernatant was dried down using nitrogen air. The precipitates were then reconstituted in a $150 \mu \mathrm{L}$ mobile phase and transferred to an autosampler vial. RDV was measured using UPLC-MS/MS, and its concentrations in ocular samples were calculated.

A Waters Acquity I-class UPLC system (Waters, Milford, MA, USA) combined with AB SCIEX QTRAP 6500 mass spectrometric (AB Sciex, Framingham, MA, USA) was used for the analysis. Chromatograms were obtained with an Acquity UPLC HSS T3 column (Waters, $2.1 \times 100 \mathrm{~mm}, 1.8 \mu \mathrm{m}$ ) using a gradient (Table S2). Positive electrospray ionization was used for all analyses. The column temperature was set at $40^{\circ} \mathrm{C}$. The total run time was $6.00 \mathrm{~min}$ with a flow rate of $0.4 \mathrm{~mL} / \mathrm{min}$. Multiple reaction monitoring traces were quantified as: $603.2>402.2$ for RDV and $330.1>128.0$ for ISS. All parameter settings are detailed in Tables S3 and S4. Standard calibration curves were constructed using five concentrations $(1.6,8,40,200$, and $1000 \mathrm{ng} / \mathrm{mL})$.

\subsection{Cell Culture}

HCECs were obtained from the Eye Bank of Affiliated Xiamen Eye Center of Xiamen University (Xiamen, Fujian, China). HCECs were cultured in 6\% fetal bovine-DMEM/F12 medium (Life Technologies, Waltham, MA, USA), containing $10 \mathrm{ng} / \mathrm{mL}$ human epidermal growth factor (Peprotech, East Windsor, NJ, USA), $10 \mu \mathrm{g} / \mathrm{mL}$ insulin (Sigma-Aldrich, USA), and 100 units/mL penicillin/streptomycin antibiotics (HyClone, Logan, UT, USA). Cells were then seeded on plastic dishes and incubated in a Galaxy $170 \mathrm{SCO}_{2}$ incubator (Eppendorf, Hamburg, Germany).

\subsection{Evaluation of Cytotoxicity}

To evaluate cytotoxicity, RDV hydrogels were extracted using $5 \mathrm{~mL}$ of DMEM/F12 medium to obtain a standard leach liquor [25]. The HCECs were seeded onto a 96-well plate with $1 \times 10^{4}$ cells per well and then co-cultured with $100 \mu \mathrm{L}$ leaching liquor. The morphological appearance of HCECs was observed using a DM IL LED microscopy (Leica, Germany) to obtain a sense of immediacy. Cell proliferation capacity was measured following the instructions of the Cell Counting Kit-8 (https:/ / www.dojindo.eu.com/TechnicalManual/ Manual_CK04.pdf, accessed on 12 December 2021, CCK-8, Dojindo, Japan).

The rate of apoptosis in HCECs was measured in all groups using the TUNEL assay. This assay identified DNA breaks by labeling the $3^{\prime}-\mathrm{OH}$ terminals of DNA with modified nucleotides to TdT or terminal nucleotidyltransferase. Briefly, HCECs were cultured on slides and then treated with the leach liquor. After an incubation period of $48 \mathrm{~h}$, the existence of apoptotic cells in the samples was investigated using a DeadEnd ${ }^{\mathrm{TM}}$ Fluorometric TUNEL System (Promega, Madison, WI, USA) in accordance with the protocol of the manufacturer.

\subsection{Evaluation of Rabbit Eye Irritation}

First, $100 \mu \mathrm{L}$ hydrogels of $1 \mathrm{mg} / \mathrm{mL}$ and $2 \mathrm{mg} / \mathrm{mL}$ were administrated topically to the left eyes of rabbits, while the right eyes served as the negative control. The left eyes were then observed with a SL9900 Digital LED slit lamp (CSO Elite, Toronto, ON, Canada) 
during the entire study period. To assess the adverse effects, intra-ocular pressure (IOP) was obtained using an iCare TV02 tonometer (iCare Finland Oy, Vantaa, Finland) between $5 \mathrm{~min}$ and $2 \mathrm{~h}$ after topical hydrogel administration. IOP was monitored five times in the left eyes of each rabbit and the median was selected for statistical analysis.

New Zealand rabbits (male, aged 3-4 months) were provided by the Experimental Animal Center of Xiamen University. The animal experiment in rabbits complied with the Association for Research in Vision and Ophthalmology Statement for the Use of Animals in Ophthalmic and Vision Research and was approved by the Experimental Animal Ethics Committee of Xiamen University (IACUC: xmulac20190063).

\subsection{Histological Analysis}

After the irritation test was completed, H\&E staining was performed to investigate the safety of the gelling in vivo. Corneas were carefully dissected and washed twice with physiological saline. The samples were then fixed in $4 \%$ formaldehyde. The fixed samples were dehydrated using different gradient concentrations of alcohol. Then, ocular tissues were embedded in paraplast plus and cut into $5-\mu \mathrm{m}$-thick sections. Histological sections were fixed in $4 \%$ paraformaldehyde, and then stained using the KeyGEN H\&E staining kit according to the kit instructions.

\subsection{Assay of Surface Plasmon Resonance}

Surface plasmon resonance (SPR) assays were performed using a Biacore T200 system and an SPR Chip CM5 of GE Healthcare (Marlborough, MA, USA) as the manual of protocols. Samples, over a range of $0.2-5.0 \mu \mathrm{mol} / \mathrm{L}$, were injected with PBS containing $5 \%$ $\operatorname{DMSO}(v / v)$ at a flow rate of $30 \mu \mathrm{L} / \mathrm{min}$. Each injection was done with $120 \mathrm{~s}$ association and dissociation time. Then, the binding activity of the hydrogel and vehicle to the spike protein of SARS-CoV-2 was investigated. $K_{\mathrm{D}}$ values were calculated using a steady affinity state model with the BIAcore T200 analysis software of GE Healthcare.

\section{Results and Discussion}

\subsection{Synthesis and Characterization of Poly(DHSe/PEG/PPG Urethane)}

Multi-block copolymers of poly(DHSe/PEG/PPG urethane) were successfully synthesized by the condensation of selenide-containing diols with PEG and PPG oligomers under modified conditions and were characterized using ${ }^{1} \mathrm{H}$ NMR (Figure 1). The synthesis process (Figure 1A) was carried out under modified conditions, with excess HDMI as the coupling reagent and dibutyltin dilaurate as the catalyzer. The poly(DHSe/PEG/PPG urethane) structure was determined by ${ }^{1} \mathrm{H}$ NMR spectroscopy (Figure 1C). For a more detailed description, the $\mathrm{CH}_{2}$ component peak of PEG was located at $3.64 \mathrm{ppm}$, and proton signals of PPG were located at 3.56-3.39 ppm, while overlapped peaks at 1.14-1.22 ppm were assigned to middle $-\mathrm{CH}_{2}$ of Se monomer. The $\mathrm{CDCl}_{3}$ proton peak $(7.26 \mathrm{ppm})$ was used as a reference. The molecular weights and dispersities $\left(\bigoplus_{\mathrm{M}}=M_{\mathrm{W}} / M_{\mathrm{n}}\right)$ of the copolymers are tabulated in Table 1.

Table 1. Molecular characteristics and thermal properties of poly(DHSe/PEG/PPG urethane).

\begin{tabular}{|c|c|c|c|c|c|c|c|}
\hline \multirow{2}{*}{ Sample } & \multicolumn{2}{|c|}{ Feed Ratio } & \multicolumn{3}{|c|}{ Thermal Analysis } & \multicolumn{2}{|c|}{ GPC } \\
\hline & PEG & PPG & $T_{\mathrm{m}}{ }^{a /{ }^{\circ} \mathrm{C}}$ & $T_{\mathrm{g}} a /{ }^{\circ} \mathrm{C}$ & $T_{\mathrm{d}}{ }^{b} /{ }^{\circ} \mathrm{C}$ & $M_{\mathrm{n}} / \mathrm{Da}$ & 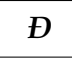 \\
\hline DHSe-11 & 1 & 1 & 41.85 & -57.11 & 298 & 32,018 & 1.14 \\
\hline DHSe-31 & 3 & 1 & 43.26 & -55.77 & 332 & 25,523 & 1.09 \\
\hline
\end{tabular}


(A)

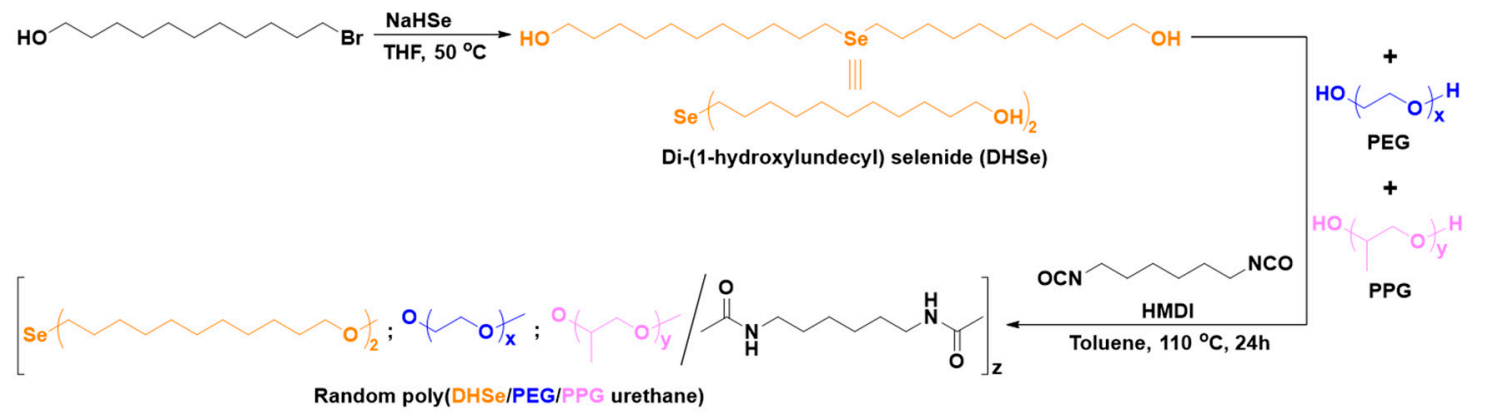

(B)
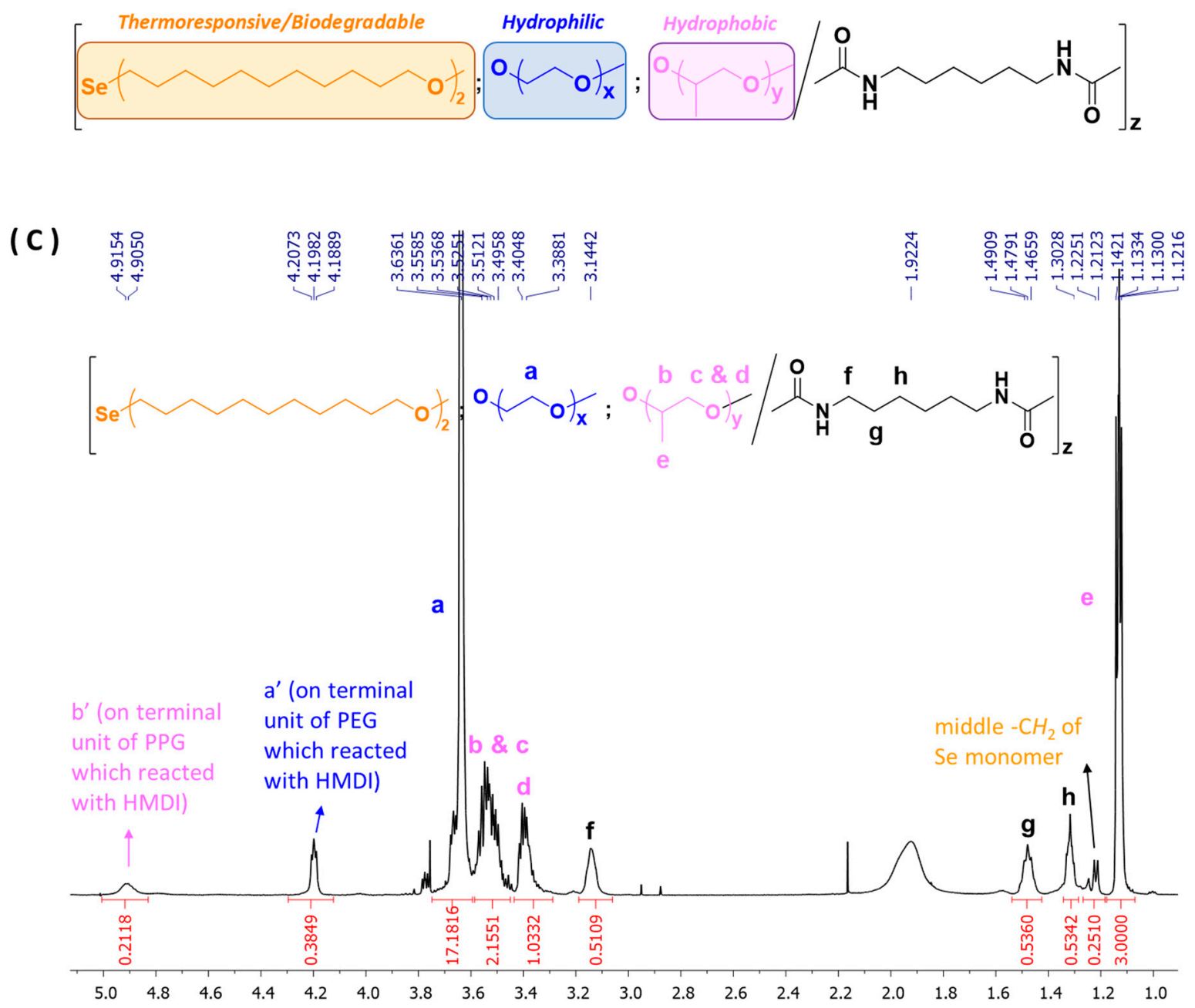

Figure 1. Synthesis and characterization of poly(DHSe/PEG/PPG urethane): (A) synthetic scheme; $(B, C)$ structure and ${ }^{1} \mathrm{H}$ NMR spectra of copolymer.

\subsection{Sol-Gel Phase Transition}

Sol-gel phase transition behavior was inspected using an inverting vial method (Figure 2A) and a stirrer method (Figure 2B). From Figure 2C, gelation occurred for the copolymer when polymer concentrations increased to a specific range; the GT decreased with copolymer concentration increase. The poly(DHSe/PEG/PPG urethane) performed reversible sol-gel transition within a $2-20 w / v \%$ concentration range. Furthermore, the gels were stable up to a higher temperature. An important advantage of poly(DHSe/PEG/PPG urethane) is the ease of optimizing the GT for different applications by altering its concentration flexibly. The MGC of poly(DHSe/PEG/PPG urethane) copolymers was approximately 
$3 w / v \%$ to accommodate to ophthalmic drug delivery. At $3 w / v \%$ concentration, the triblock copolymer can form a solution state at room temperature and gel at the temperature of the ocular surface, which is favorable for the topical ocular delivery of RDV. The thermogels described in the remaining part of this paper are the $3 w / v \%$ concentration poly(DHSe/PEG/PPG urethane).

A
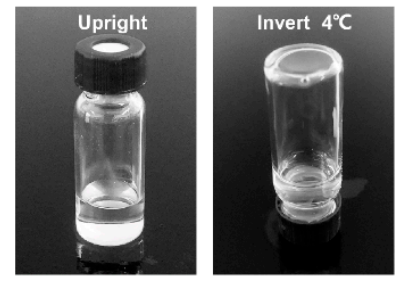

C

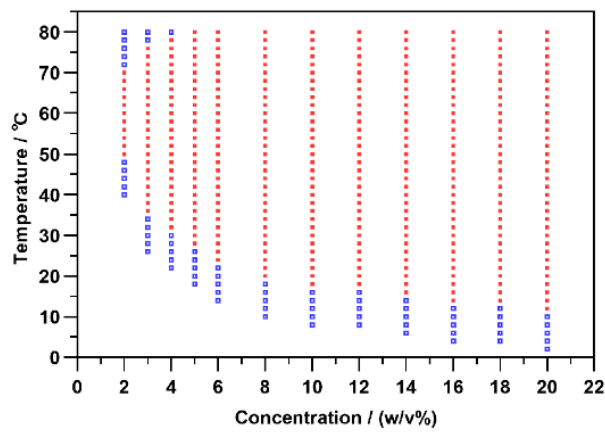

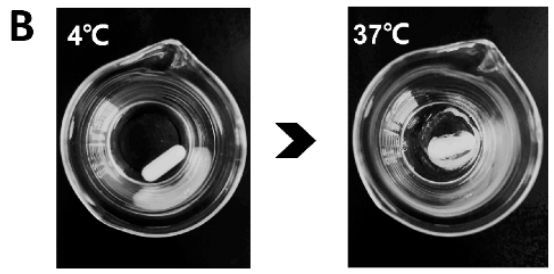

D

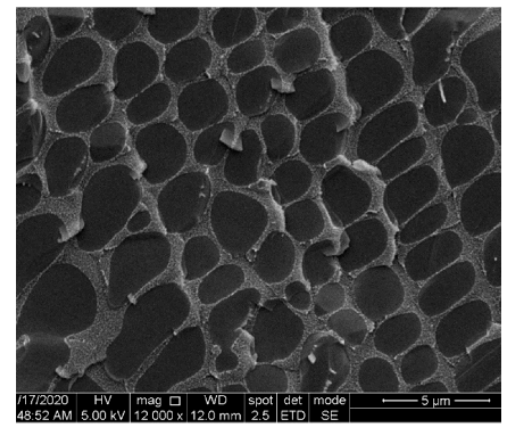

Figure 2. Sol-gel transition was tested by an inverting vial method (A) and a stirrer method (B); (C) phase diagrams of 2-20 w/v\% copolymer aqueous solution (" $\square$ " gelation occurred, " $\square$ " solution condition); (D) Cryo-SEM image of the copolymer.

Additionally, polymerized materials with high moisture content were prone to lose water in vacuum, rendering the structural characterization difficult to perform [26]. In this paper, Cryo-SEM with technical assistance by a cooling stage and a liquid nitrogen pool (quick freeze so ice crystals did not have as much time to form) was applied to confirm the morphological results for the hydrogels. The porous structure of the hydrogel was confirmed with a pore size range of 2 to $10 \mu \mathrm{m}$ (Figure 2D).

\subsection{Thermal and Rheological Properties}

The copolymers exhibited a favorable thermal characteristic. Only one $T_{\mathrm{g}}$ value was observed, indicating DHSe/PEG/PPG miscibility (Figure 3A,B and Table 1). TGA and DSC studies showed that DHSe-11 and DHSe-31 hydrogels were thermally stable. The poly(DHSe/PEG/PPG urethane) was further characterized by rheological methods. The oscillation dependence of the gels was tested; DHSe-11 hydrogel breaks under high strain, while the DHSe-31 hydrogel remained stable in the whole range of the tested strain (Figure 3C). In the frequency dependence test (Figure 3D), mechanical properties increased linearly with frequency. The thermogel was stable throughout the experiment, with $G^{\prime}>G^{\prime \prime}$. Both hydrogels are stable in the tested range of frequency. According to the above results, the DHSe-31 hydrogel showed a higher modulus in both the amplitude and frequency sweep test; thus, the feed ratio of PEG and PPG oligomers was set at 3:1. 
(A)

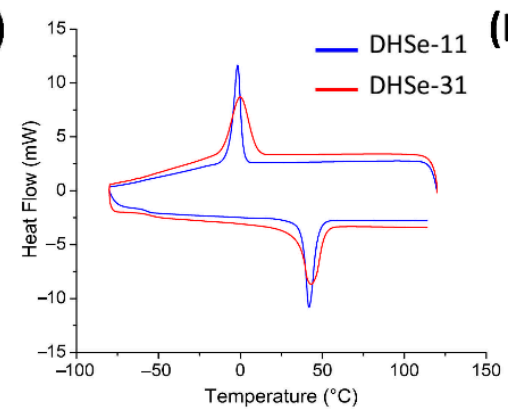

(C)

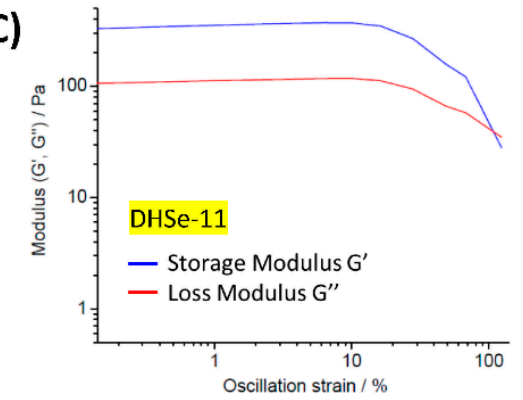

(D)

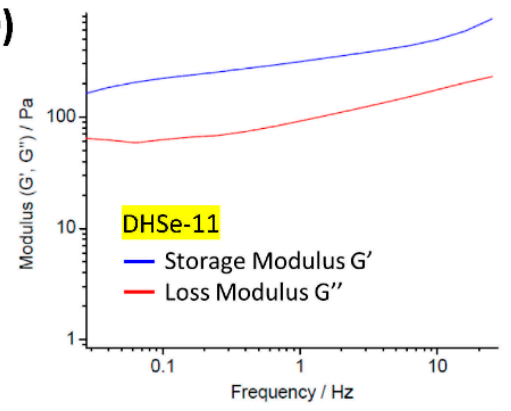

(B)
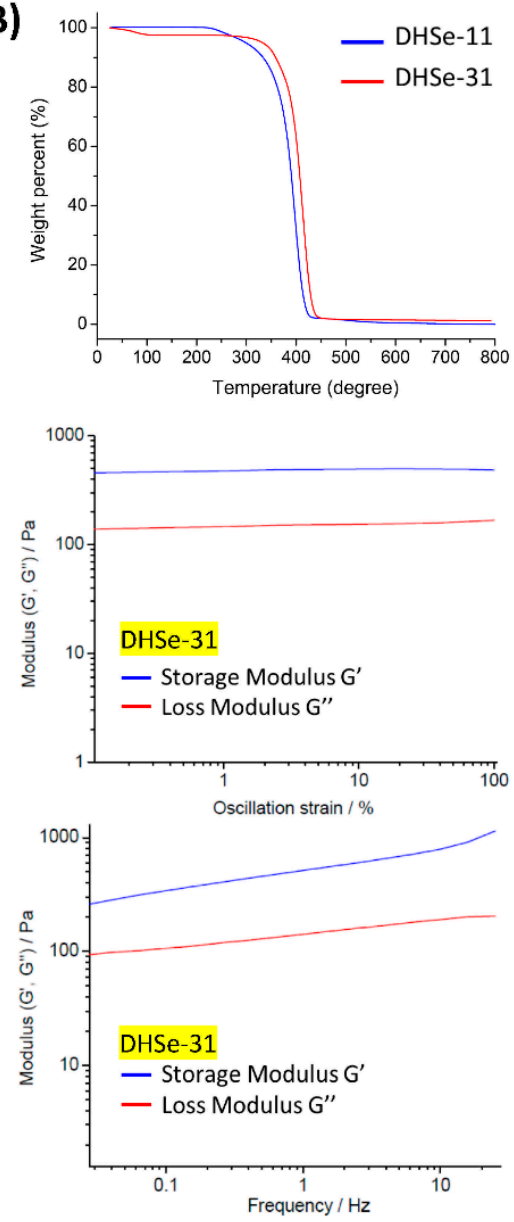

Figure 3. Thermal and rheological properties of hydrogels: (A,B) DSC and TGA results; (C) oscillatory strain sweep plots; (D) frequency sweep of DHSe-11 and DHSe-31 hydrogel.

\subsection{Transparency of RDV/Poly(DHSe/PEG/PPG Urethane) Hydrogel}

As an important property of ophthalmic biomaterials, biomedical materials exhibiting transparency of over $90 \%$ versus visible wavelengths are considered to be highly transmissive. Through the macroscopic examination of a letter " $\mathrm{A}$ " card, the clarity of the RDV/poly(DH-Se/PEG/PPG urethane) hydrogel was macroscopically observed (Figure 4A). The RDV/poly(DHSe/PEG/PPG urethane) hydrogel showed clearer visibility when compared with the RDV-loaded poloxamer hydrogel. Absorbance curves in the visible region were also analyzed to perform a thorough and quantitative evaluation (Figure 4B). Transmittance curves at different wavelengths were further calculated (Table S1, Figure 4C,D). It can be clearly seen that the poloxamer hydrogel had poorer transmittance performance compared with RDV/poly(DHSe/PEG/PPG urethane).

\subsection{In Vitro RDV Release Behavior}

To further demonstrate the feasibility of the polyurethane-based thermogel as an RDV carrier, a drug release study was performed. The release profile showed that the cyclodextrin inclusion system exhibited the rapid release of RDV within $1 \mathrm{~h}$ (Figure 5). Conversely, it could be seen that the thermogel provided the sustained/prolonged release of $80 \% \mathrm{RDV}$ in $4 \mathrm{~h}$. The slow releasing of RDV might be attributed to the presence of a reticular structure inside the poly(DHSe/PEG/PPG urethane) hydrogel. Moreover, the in vitro RDV release behavior conformed to the diffusion-based dissolution model [18,20]. 
(A) ddW Pluronic Polyurethane

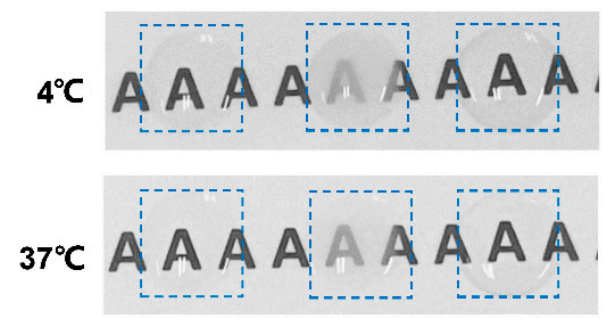

(C)

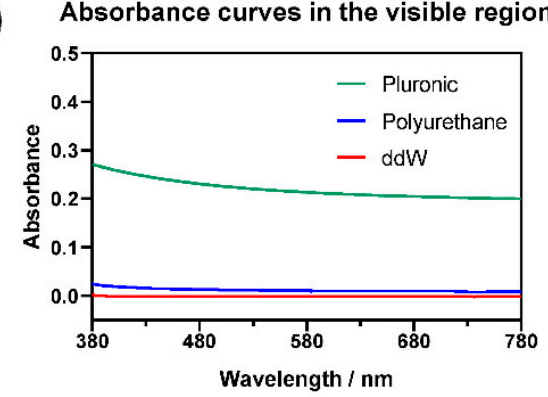

(B) Transparency curves in the visible region

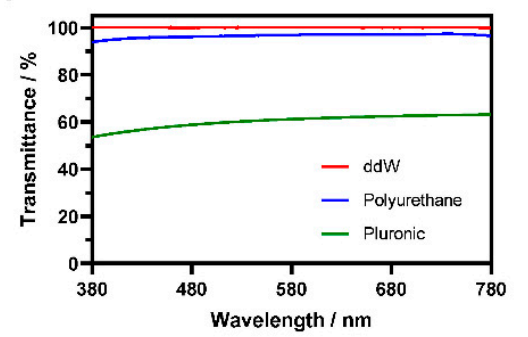

(D)

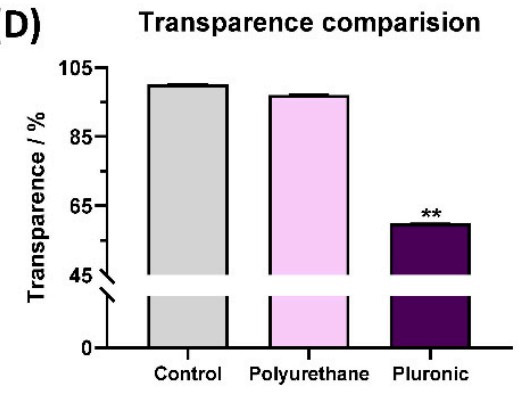

Figure 4. Optical properties of hydrogels: (A) macroscopic evaluation of transmittance performance; $(\mathbf{B}, \mathbf{C})$ transmittance and absorbance curves versus visible wavelength; (D) transmittance comparison $(* * p<0.01)$.

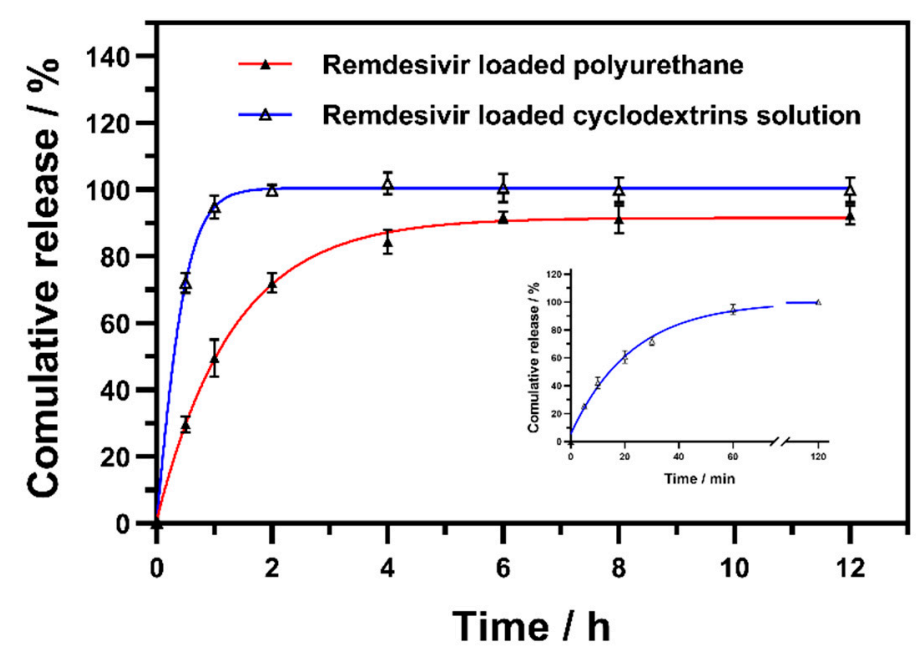

Figure 5. In vitro RDV release profiles of thermogel and cyclodextrin inclusion complex in artificial tears.

\subsection{Drug Retention and Penetration Test}

The retention time is an important characteristic of eye drops. As shown in Figure 6A, the fluorescein sodium retention was extended to around $60 \mathrm{~min}$ by introducing poly(DHSe/PEG/PPG urethane). Given the universal quenching rate of the fluorescent probe, the accurate prolonged retention time might be relatively longer than $60 \mathrm{~min}$. Conversely, the fluorescein sodium clearance was rapid in the cyclodextrin inclusion compound, and fluorescence staining was almost invisible at $15 \mathrm{~min}$. Compared with a chitosan-based hydrogel [21], poly(DHSe/PEG/PPG urethane) provided a relatively longer-term precorneal retention time (over $60 \mathrm{~min}$ vs. $30 \mathrm{~min}$ ), thus effectively resisting the effect of tear drainage.

OCT was applied to visualize RDV retention behavior. PBS was used to simulate the precorneal retention behavior of commonly used clinical eye drops. A hydration film, structured in close proximity to the corneal surface, was formed immediately after topical 
PBS administration and was readily cleared due to blinking of the eyelids (Figure 6B). The cyclodextrin inclusion complex aqueous solution, which should behave as a viscous liquid, exhibited an uneven hydration film as with PBS, and was sustained for a short time (Figure 6C). In contrast, rapid gelation of the poly(DHSe/PEG/PPG urethane) was observed and resulted in a thick, well-distributed coating on the cornea (Figure 6D). The gelation layer remained on the ocular surface after $50 \mathrm{~min}$. This result was in agreement with the result of the fluorescein sodium retention test.

(A)

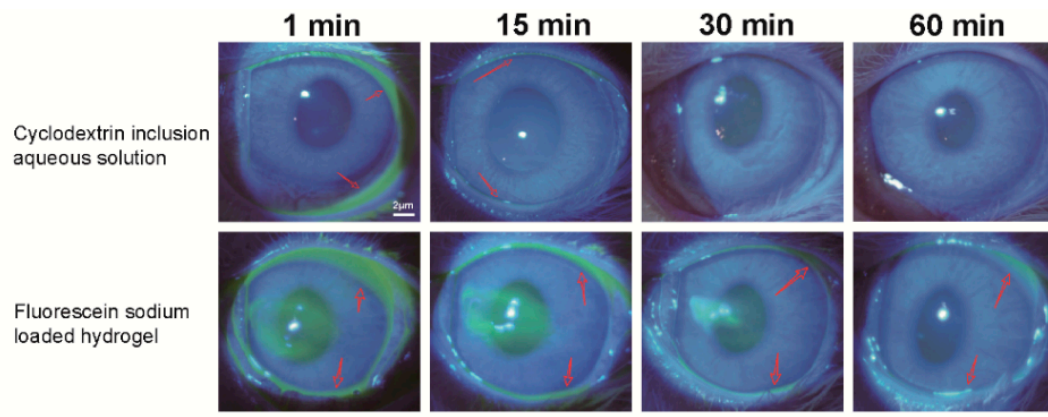

(B)

$$
\text { Baseline }
$$

$5 \mathrm{~s}$

$30 \mathrm{~s}$
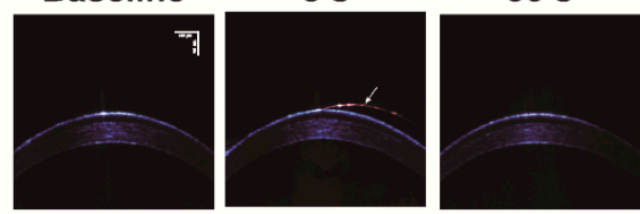

$5 \mathrm{~min}$

$50 \mathrm{~min}$

(C)
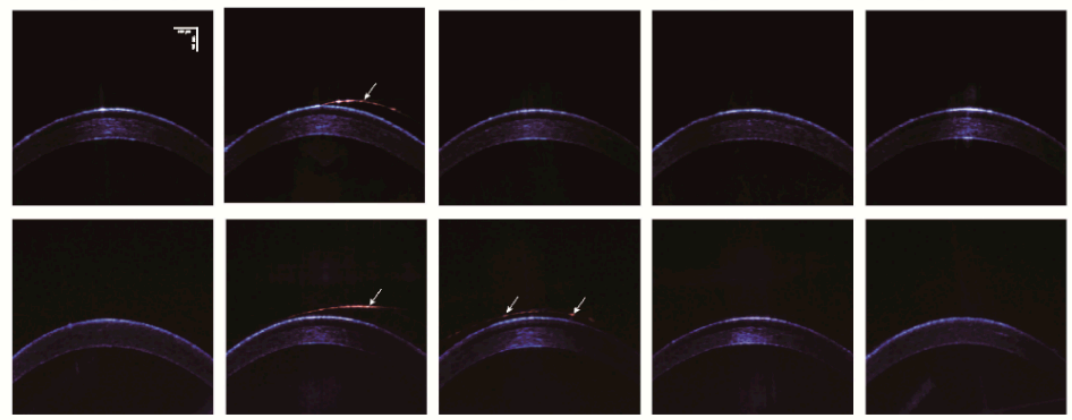

(D)
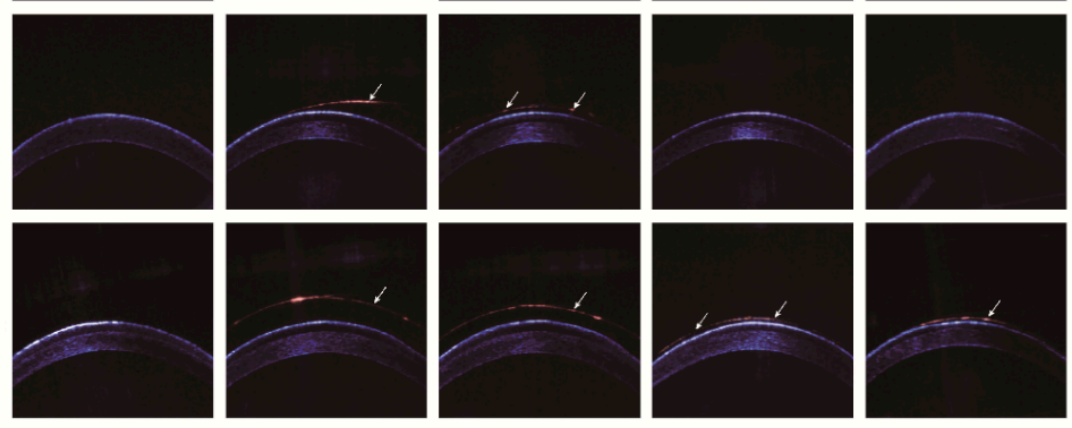

(E)
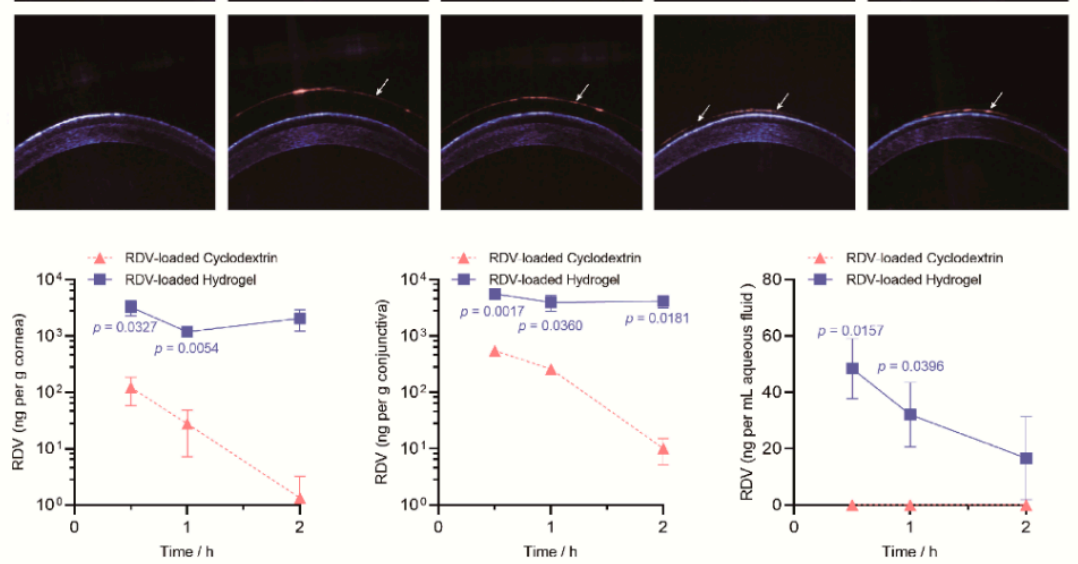

Figure 6. Precorneal retention and RDV penetration results: (A) fluorescent dye test; (B) PBS; (C) cyclodextrin inclusion compound; (D) hydrogel retention behavior using OCT imaging; (E) RDV concentration in ocular tissues after instillation.

UPLC-MS/MS was used to calculate the concentration of RDV penetration in ocular tissues. The retention times were $3.31 \mathrm{~min}$ for RDV and $2.59 \mathrm{~min}$ for ISS (Figure S1). The linearity range for RDV was from $1.6 \mathrm{ng} / \mathrm{mL}$ to $1000 \mathrm{ng} / \mathrm{mL}$, while the limit of detection was $0.97 \mathrm{ng} / \mathrm{mL}$ (Figure S1). RDV in the poly(DHSe/PEG/PPG urethane) hydrogel provided significantly higher drug concentrations in rabbit corneas ( 0.5 and $1.0 \mathrm{~h}$ ) compared with the cyclodextrin inclusion compound (Figure 6E). We found a similar trend in the conjunctiva, and the greater concentrations of RDV were significant at all points. The RDV in the cyclodextrin inclusion compound solution was indetectable in the aqueous fluid. Despite the low concentration (several tens of nanograms), we found a consistent increase in the RDV/poly(DHSe/PEG/PPG urethane) hydrogel formulation. 
We found that poly(DHSe/PEG/PPG urethane) effectively enhanced RDV permeation into the anterior ganglion tissue more than that of cyclodextrin. The prolonged RDV retention on the ocular surface is a possible reason for this result.

\subsection{In Vitro Cytotoxicity}

In general, the cell morphology results showed that the structure and density of HCECs treated with leach liquor were normal (Figure S2). Results of the TUNEL assay and fluorescence intensity analysis are shown in Figure 7A,B. There was a significant difference between the normal group and the positive control (treated with DNase I) or the negative control, in terms of apoptosis measured with TUNEL staining $(p<0.01)$. Compared with the normal group, no significant differences were observed after treatment with leach liquor, according to the fluorescence intensity Arbitrary Unit (A.U.) analysis. As shown in Figure 7C, the results of the CCK-8 assay on HCECs indicated that RDV/poly(DHSe/PEG/PPG urethane caused minimal cytotoxicity (cell viability $>90 \%$ ) for HCECs after $72 \mathrm{~h}$ of incubation. This result suggests that the thermogel system might act as a safe vehicle for ophthalmic drug delivery.

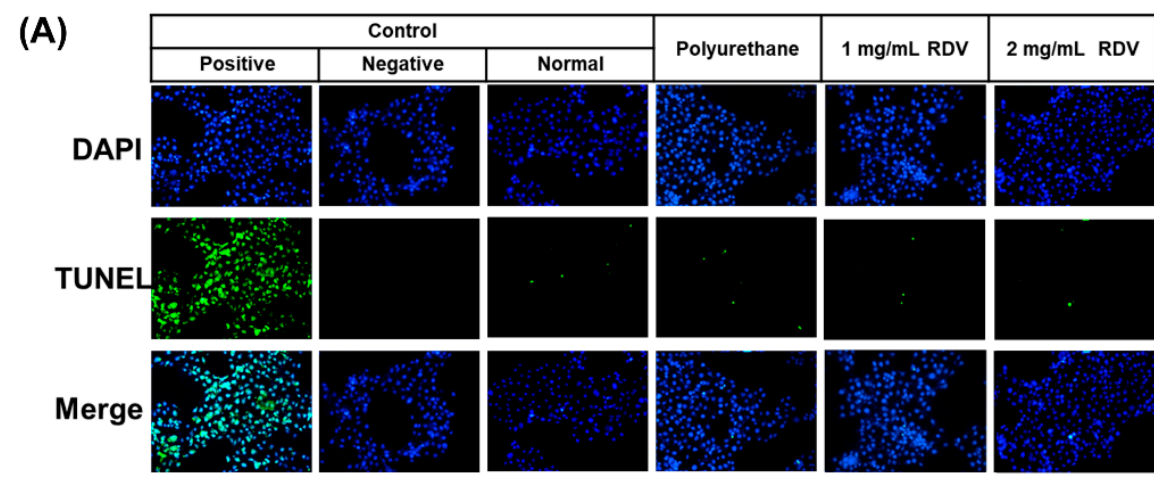

(B) Fluorescence intensity analysis
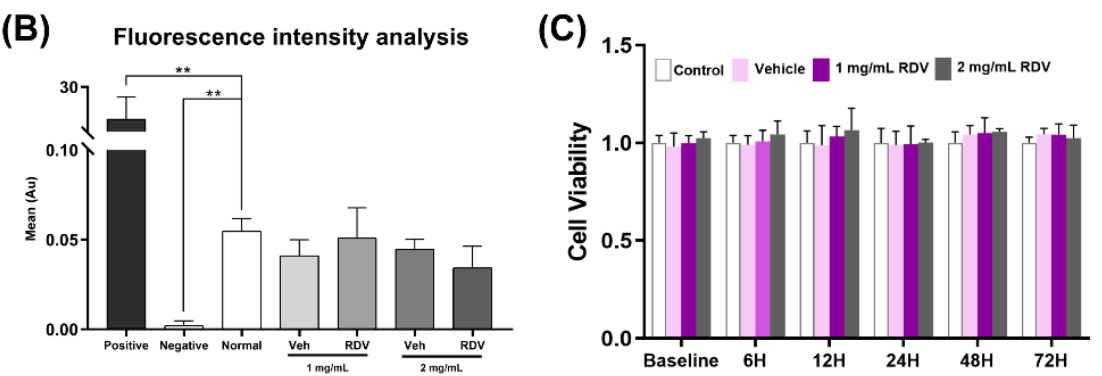

Figure 7. In vitro cytotoxicity result: (A) TUNEL staining of HCECs after different treatments; (B) fluorescence intensity analysis of TUNEL assay (mean $\pm \mathrm{SD},{ }^{* *} p<0.01$ vs. normal); (C) proliferation ability assay by CCK-8.

\subsection{Ocular Irritation and Histological Evaluation}

After a single topical administration of the RDV/poly(DHSe/PEG/PPG urethane) hydrogel and its carrier, no signs of conjunctival congestion and edema, corneal epithelial exfoliation, or opacification appeared in all groups, suggesting that the hydrogels were well-tolerated (Figure 8A,B). Post-drop application, IOP in rabbits treated with hydrogels remained stable during the test period (one-way ANOVA test, control group vs. $1 \mathrm{mg} / \mathrm{mL}$ RDV hydrogel group ( $p=0.0516)$, and control group vs. $2 \mathrm{mg} / \mathrm{mL}$ RDV hydrogel group $(p=0.3307))$. In the eyes treated with the carrier, the mean IOP varied widely, with a low average, but no significant difference was observed (Figure 8E). The results of IOP demonstrated that poly(DHSe/PEG/PPG urethane) showed no IOP-lowering effect after administration for $2 \mathrm{~h}$, and the change in IOP was also more stable than poloxamer, carbopol, and PU/HPCD [20]. 

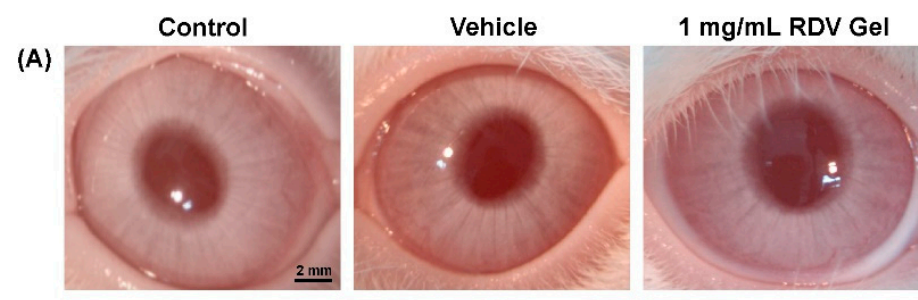

$2 \mathrm{mg} / \mathrm{mL}$ RDV Gel

(B)
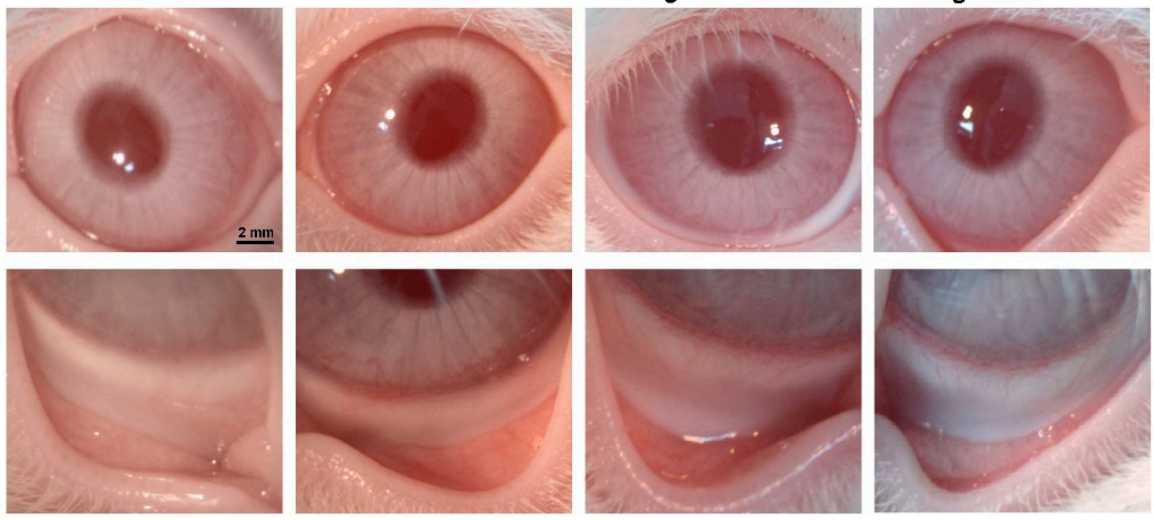

(C)
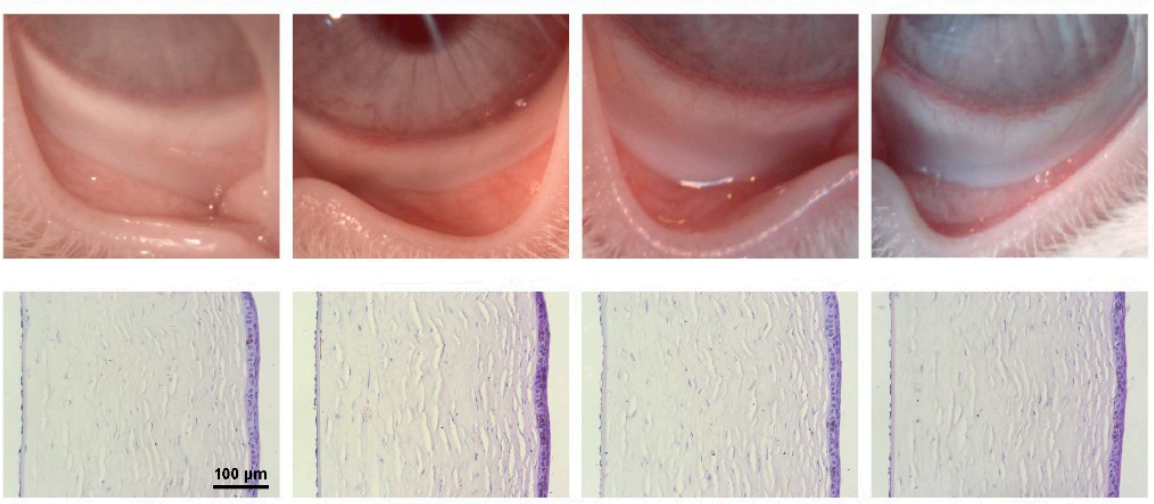

(D)
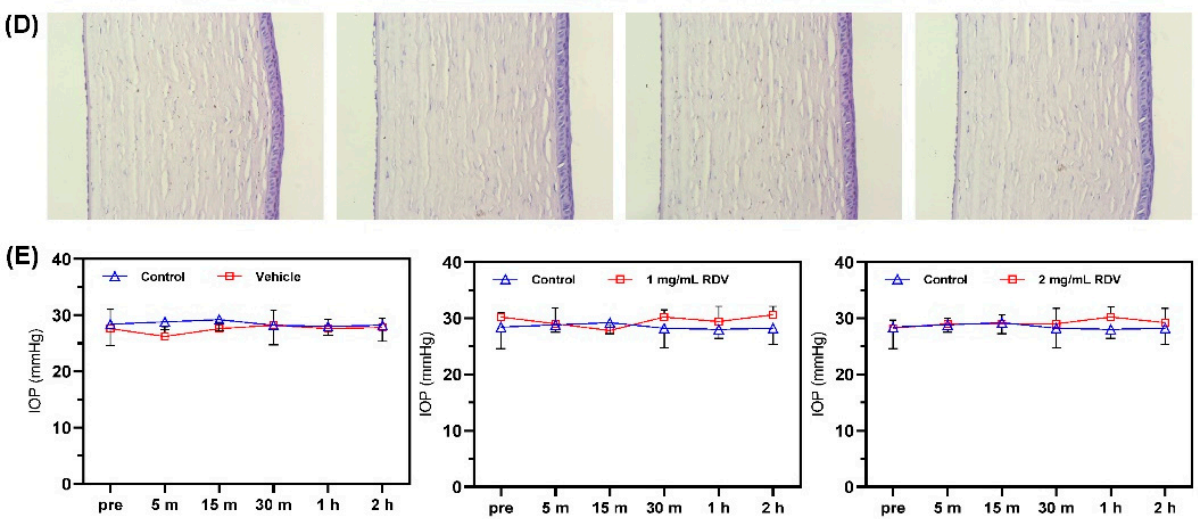

Figure 8. Ocular irritation observation and histological evaluation: (A,B) corneal and conjunctival toleration under the slit-lamp microscope; (C,D) H\&E staining of center and peripheral region of cornea; (E) IOP of rabbits after treatment.

After the irritation test, histopathological examination of the corneas was used to evaluate the hydrogels' safety. The top row of the figure illustrates the central cornea (Figure 8C), and the following row illustrates the peripheral region of the cornea (Figure 8D). After ocular administration, corneal cells possessed a clear outline and were tightly arranged, without epithelial defects and inflammatory cell infiltration. H\&E sections of corneas revealed normal epithelium linings and corneal stroma, including the corneal tissues of rabbits that received the hydrogel vehicle and RDV-containing hydrogel. Thus, the hydrogel was well-biocompatible for rabbit eyes.

\subsection{SPR Analysis with Spike Glycoprotein}

The binding affinities of RDV and spike glycoprotein were verified in vitro. As shown in Figure 9, the biosensor responses to the vehicle were significantly low due to the nonspecific interaction with the spike protein $\left(K_{\mathrm{D}}=2.307 \mathrm{mM}\right.$, Figure $\left.9 \mathrm{~A}\right)$. However, the interactions between the hydrogel and the SARS-CoV-2 spike protein were concentrationdependent. The $K_{\mathrm{D}}$ value between RDV and spike glycoprotein was $28.60 \mathrm{nmol} / \mathrm{L}$ (Figure 9B), indicating a strong affinity. The response curve maintained a good affinity $\left(K_{\mathrm{D}}=37.18 \mathrm{nM}\right)$ after RDV with loaded with polyurethane (Figure $\left.9 \mathrm{C}\right)$. These results suggest that using an RDV hydrogel is an efficient strategy for the prevention of SARSCoV-2 infection, given that affinity was not blocked by the hydrogel carrier. Meanwhile, this result indicates a potential pathway of RDV preventing SARS-CoV-2 transmission, 
not only through inhibiting RNA polymerase. Follow-up studies are required to verify this result.
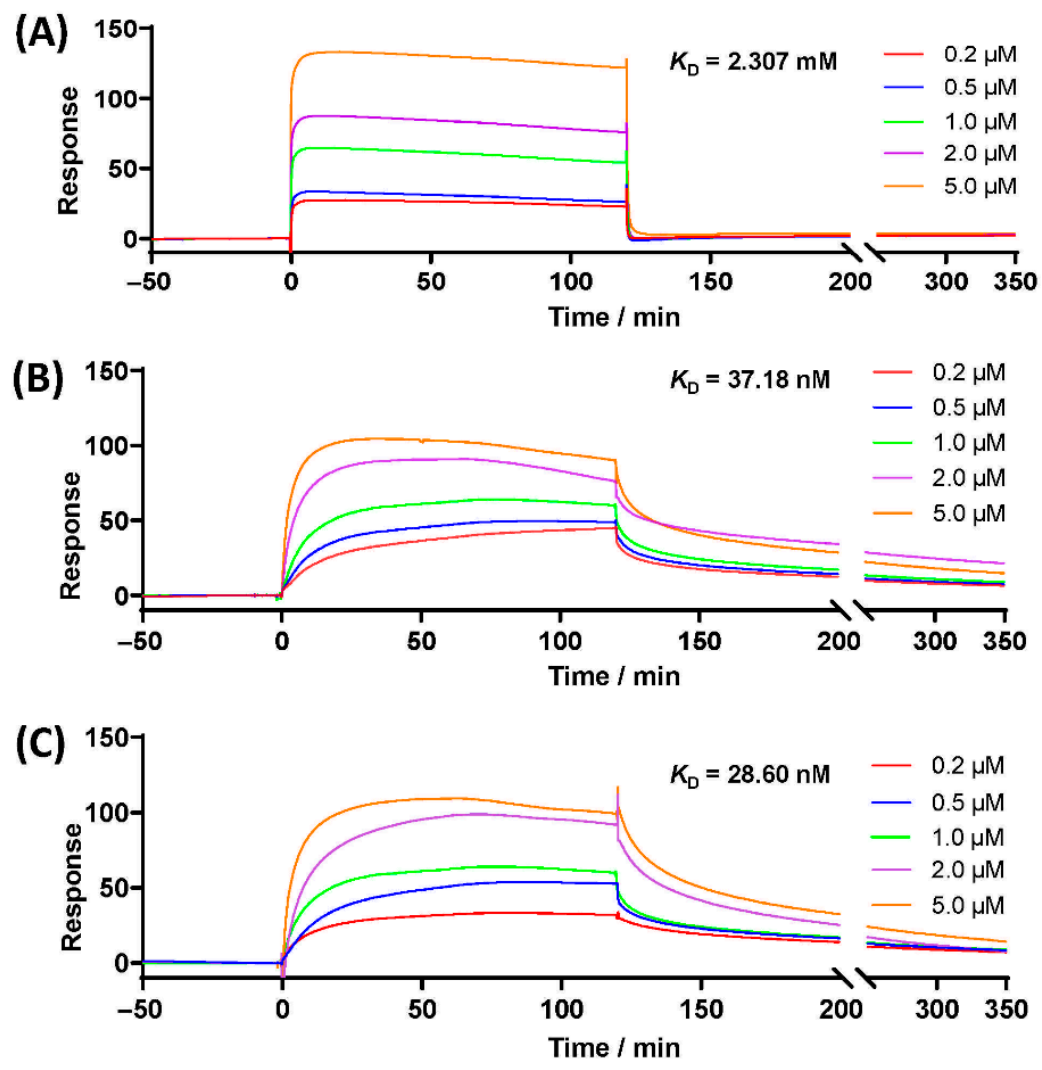

Figure 9. SPR biosensor analysis: (A) poor affinity between vehicle and spike protein $\left(K_{\mathrm{D}}=2.307 \mathrm{mM}\right) ;(\mathbf{B})$ favorable binding of RDV and spike protein $\left(K_{\mathrm{D}}=37.18 \mathrm{nM}\right)$; $(\mathbf{C})$ the binding would not be blocked by hydrogel carrier $\left(K_{\mathrm{D}}=28.60 \mathrm{nM}\right)$.

\section{Conclusions}

In this work, an RDV-loaded poly (DHSe/PEG/PPG urethane) thermogel was successfully developed as a topical ocular delivery system. Compared with the previously reported triblock copolymer, the proposed poly(DHSe/PEG/PPG urethane) hydrogel formed was prepared at different feed ratios of PEG and PPG. The RDV-loaded polyurethane hydrogel was prepared using a two-step method in a low-temperature environment. The polyurethane thermogel had a typical sol-gel transition at $35^{\circ} \mathrm{C}$ and showed favorable optical, rheological, and thermal properties. More importantly, the RDV/poly(DHSe/PEG/PPG urethane) hydrogel system at an eye drop dose caused minimal toxicity against HCECs and rabbit eyes. To the best of our knowledge, this is the first attempt to develop a drug delivery system using RDV loaded into a hydrogel. Compared with aqueous solution formulation, the RDV/poly(DHSe/PEG/PPG urethane) hydrogel significantly enhanced the ocular retention and penetration, thus providing a potential method to block SARS-CoV-2 transmission via the ocular surface.

Supplementary Materials: The following are available online at https:/ / www.mdpi.com/article/10 .3390/pharmaceutics14010050/s1, Figure S1: (A) The retention times for RDV and ISS; (B) linearity range of RDV, Figure S2: Morphological appearance of HCECs at different magnifications, Table S1: The transparence of three systems at different wavelengths (\%), Table S2: Chromatographic gradient, Table S3: General instrument settings, Table S4: Analyte-specific parameters. 
Author Contributions: Conceptualization, Y.Q. and J.R.; methodology, S.X. and L.K.; software, S.Z.; validation, S.X., Y.X. and Z.L.; formal analysis, L.K. and Y.W.; investigation, Y.W.; resources, Y.Q. and J.R.; supervision, Y.Q.; project administration, S.X.; funding acquisition, Y.Q. All authors have read and agreed to the published version of the manuscript.

Funding: This work was supported by funds from the Fundamental Research Funds for the Central Universities (No. 20720200015), Fujian Health-Education Research Grant (No. WKJ2016-2-03), and XMU Training Program of Innovation and Entrepreneurship for Undergraduates (No. 202010384289, 2020X0727, 2021X1071).

Institutional Review Board Statement: The study was conducted according to the guidelines of the Declaration of Helsinki, and approved by the Experimental Animal Ethics Committee of Xiamen University (IACUC: xmulac20190063, 14 November 2019).

Informed Consent Statement: Not applicable.

Data Availability Statement: The data presented in this study are available on request from the corresponding author.

Acknowledgments: Authors would like to acknowledge Yi Han and Zihan Chen for their superb slit-lamp operation and extensive experience. Special thanks to the medical staff for their help during the COVID-19 outbreak.

Conflicts of Interest: The authors declare that they have no known competing financial interests or personal relationships that affected the work reported in this paper.

\section{References}

1. Guo, Y.-R.; Cao, Q.-D.; Hong, Z.-S.; Tan, Y.-Y.; Chen, S.-D.; Jin, H.-J.; Tan, K.-S.; Wang, D.-Y.; Yan, Y. The origin, transmission and clinical therapies on coronavirus disease 2019 (COVID-19) outbreak-An update on the status. Mil. Med. Res. $2020,7,11$. [CrossRef] [PubMed]

2. Kesson, A. Respiratory virus infections. Paediatr. Respir. Rev. 2007, 8, 240-248. [CrossRef] [PubMed]

3. Belser, J.A.; Maines, T.R.; Tumpey, T.M.; Katz, J.M. Influenza A virus transmission: Contributing factors and clinical implications. Expert Rev. Mol. Med. 2010, 12, e39. [CrossRef] [PubMed]

4. Belser, J.; Rota, P.; Tumpey, T. Ocular tropism of respiratory viruses. Microbiol. Mol. Biol. Rev. 2013, 77, 144-156. [CrossRef]

5. Chen, X.; Yu, H.; Mei, T.; Chen, B.; Chen, L.; Li, S.; Zhang, X.; Sun, X. SARS-CoV-2 on the ocular surface: Is it truly a novel transmission route? Br. J. Ophthalmol. 2020, 105, 1190-1195. [CrossRef] [PubMed]

6. Napoli, P.; Nioi, M.; D’Aloja, E.; Fossarello, M. The Ocular Surface and the Coronavirus Disease 2019: Does a Dual 'Ocular Route' Exist? J. Clin. Med. 2020, 9, 1269. [CrossRef] [PubMed]

7. Zhou, L.; Xu, Z.; Castiglione, G.M.; Soiberman, U.S.; Eberhart, C.G.; Duh, E.J. ACE2 and TMPRSS2 are expressed on the human ocular surface, suggesting susceptibility to SARS-CoV-2 infection. Ocul. Surf. 2020, 18, 537-544. [CrossRef] [PubMed]

8. Khan, M.M.; Parab, S.R.; Paranjape, M. Repurposing 0.5\% povidone iodine solution in otorhinolaryngology practice in Covid 19 pandemic. Am. J. Otolaryngol. 2020, 41, 102618. [CrossRef] [PubMed]

9. Kronbichler, A.; Effenberger, M.; Eisenhut, M.; Lee, K.H.; Shin, J.I. Seven recommendations to rescue the patients and reduce the mortality from COVID-19 infection: An immunological point of view. Autoimmun. Rev. 2020, 19, 102570. [CrossRef]

10. Beigel, J.H.; Tomashek, K.M.; Dodd, L.E.; Mehta, A.K.; Zingman, B.S.; Kalil, A.C.; Hohmann, E.; Chu, H.Y.; Luetkemeyer, A.; Kline, S.; et al. Remdesivir for the Treatment of COVID-19_Preliminary report. N. Engl. J. Med. 2020, 383, 1813-1826. [CrossRef]

11. Choy, K.T.; Wong, A.Y.; Kaewpreedee, P.; Sia, S.F.; Chen, D.; Hui, K.P.Y.; Chu, D.K.W.; Chan, M.C.W.; Cheung, P.P.; Huang, X.; et al. Remdesivir, lopinavir, emetine, and homoharringtonine inhibit SARS-CoV-2 replication in vitro. Antivir. Res. 2020, $178,104786$. [CrossRef]

12. Choi, S.W.; Shin, J.S.; Park, S.J.; Jung, E.; Park, Y.G.; Lee, J.; Kim, S.J.; Park, H.J.; Lee, J.H.; Park, S.M.; et al. Antiviral activity and safety of remdesivir against SARS-CoV-2 infection in human pluripotent stem cell-derived cardiomyocytes. Antivir. Res. 2020, 184, 104955. [CrossRef]

13. Xie, J.; Wang, Z. Can remdesivir and its parent nucleoside GS-441524 be potential oral drugs? An in vitro and in vivo DMPK assessment. Acta Pharm. Sin. B 2021, 11, 1607-1616. [CrossRef] [PubMed]

14. Li, J.; Zhao, H.; Okeke, C.I.; Li, L.; Liu, Z.; Yin, Z.; Zhuang, P.; Sun, J.; Wu, T.; Wang, M.; et al. Comparison of systemic absorption between ofloxacin ophthalmic in situ gels and ofloxacin conventional ophthalmic solutions administration to rabbit eyes by HPLC-MS/MS. Int. J. Pharm. 2013, 450, 104-113. [CrossRef] [PubMed]

15. Higuchi, A.; Takahashi, K.; Hirashima, M.; Kawakita, T.; Tsubota, K. Selenoprotein P Controls Oxidative Stress in Cornea. PLoS ONE 2010, 5, e9911. [CrossRef] [PubMed]

16. Higuchi, A. Development of New Pharmaceutical Candidates with Antioxidant Activity for the Treatment of Corneal Disorders. Cornea 2019, 38, S45-S49. [CrossRef] [PubMed] 
17. Loh, X.J.; Tan, Y.X.; Li, Z.; Teo, L.S.; Goh, S.H.; Li, J. Biodegradable thermogelling poly(ester urethane)s consisting of poly(lactic acid)-Thermodynamics of micellization and hydrolytic degradation. Biomaterials 2008, 29, 2164-2172. [CrossRef] [PubMed]

18. Luo, Z.; Jiang, L.; Yang, S.; Li, Z.; Soh, W.M.W.; Zheng, L.; Loh, X.J.; Wu, Y. Light-Induced Redox-Responsive Smart Drug Delivery System by Using Selenium-Containing Polymer@MOF Shell/Core Nanocomposite. Adv. Heal. Mater. 2019, 8, e1900406. [CrossRef]

19. Gao, J.; Wu, S.; Emge, T.J.; Rogers, M.A. Nanoscale and microscale structural changes alter the critical gelator concentration of self-assembled fibrillar networks. CrystEngComm 2013, 15, 4507-4515. [CrossRef]

20. Qi, H.; Chen, W.; Huang, C.; Li, L.; Chen, C.; Li, W.; Wu, C. Development of a poloxamer analogs/carbopol-based in situ gelling and mucoadhesive ophthalmic delivery system for puerarin. Int. J. Pharm. 2007, 337, 178-187. [CrossRef] [PubMed]

21. Shi, H.; Wang, Y.; Bao, Z.; Lin, D.; Liu, H.; Yu, A.; Lei, L.; Li, X.; Xu, X. Thermosensitive glycol chitosan-based hydrogel as a topical ocular drug delivery system for enhanced ocular bioavailability. Int. J. Pharm. 2019, 570, 118688. [CrossRef] [PubMed]

22. Hua, S. Comparison of in vitro dialysis release methods of loperamide-encapsulated liposomal gel for topical drug delivery. Int. J. Nanomed. 2014, 9, 735-744. [CrossRef] [PubMed]

23. Han, Y.; Xu, C.; Shi, H.; Yu, F.; Zhong, Y.; Liu, Z.; Loh, X.J.; Wu, Y.-L.; Li, Z.; Li, C. Engineered bio-adhesive polyhedral oligomeric silsesquioxane hybrid nanoformulation of amphotericin B for prolonged therapy of fungal keratitis. Chem. Eng. J. 2021, 421, 129734. [CrossRef]

24. Avataneo, V.; de Nicolò, A.; Cusato, J.; Antonucci, M.; Manca, A.; Palermiti, A.; Waitt, C.; Walimbwa, S.; Lamorde, M.; di Perri, G.; et al. Development and validation of a UHPLC-MS/MS method for quantification of the prodrug remdesivir and its metabolite GS-441524: A tool for clinical pharmacokinetics of SARS-CoV-2/COVID-19 and Ebola virus disease. J. Antimicrob. Chemother. 2020, 75, 1772-1777. [CrossRef]

25. Liu, B.; Zhao, Y.; Zhu, T.; Gao, S.; Ye, K.; Zhou, F.; Qiu, D.; Wang, X.; Tian, Y.; Qu, X. Biphasic Double-Network Hydrogel With Compartmentalized Loading of Bioactive Glass for Osteochondral Defect Repair. Front. Bioeng. Biotechnol. 2020, 8, 752. [CrossRef] [PubMed]

26. Aston, R.; Sewell, K.; Klein, T.; Lawrie, G.; Grøndahl, L. Evaluation of the impact of freezing preparation techniques on the characterisation of alginate hydrogels by cryo-SEM. Eur. Polym. J. 2016, 82, 1-15. [CrossRef] 